\title{
The Effect of Family Psychoeducation on Burden of Care in Families with Type I Diabetic Children
}

\author{
Behnam Farahmandnia, ${ }^{1}$ Bahman Dashtbozorgi, ${ }^{2,}{ }^{*}$ Hooshang Alijani Renani, ${ }^{3}$ Seyed Mahmoud \\ Latifi, $^{4}$ and Majid Aminzadeh ${ }^{5}$ \\ ${ }^{1}$ Student Research Committee, Ahvaz Jundishapur University of Medical Sciences, Ahvaz, Iran \\ ${ }^{2}$ Nursing Care Research Center in Chronic Diseases, School of Nursing \& Midwifery, Ahvaz Jundishapur University of Medical Sciences, Ahvaz, Iran \\ ${ }^{3}$ School of Nursing and Midwifery, Ahvaz Jundishapur University of Medical Sciences, Ahvaz, Iran \\ ${ }^{4}$ Diabetes Research Center, Ahvaz Jundishapur University of Medical Sciences, Ahvaz, Iran \\ ${ }^{5}$ School of Medicine, Ahvaz Jundishapur University of Medical Sciences, Ahvaz, Iran \\ "Corresponding author: Bahman Dashtbozorgi, Nursing Care Research Center in Chronic Diseases, School of Nursing \& Midwifery, Ahvaz Jundishapur University of Medical \\ Sciences, Ahvaz, Iran. Tel: +98-9163134981, Fax: +98-6133738333, E-mail: bahnurse@ajums.ac.ir
}

Received 2017 May 08; Accepted 2017 June 26.

\begin{abstract}
Background: When controlling diabetes in children, families suffer from burden of care imposed by the disease. The aim of this study was to investigate the effect of family psychoeducation on the burden of care imposed on families of adolescents with type 1 diabetes referring to Golestan diabetes clinic in Ahvaz city.

Methods: The present study was a clinical trial with before-after design. The study population included families with adolescents with type 1 diabetes aged 12 - 19 years. A total of 40 families of sick children and one parent who was more in touch with the patient were selected and randomly divided into experimental (20 families) and control groups ( 20 families). To measure family burden of care, burden of care Questionnaire (DFI-S) was used. Family psychoeducation was carried out for the experimental group twice a week for 8 sessions of 90 minutes. Independent and paired t-test and Mann-Whitney test were used to compare the mean score of family burden of care in both groups while Chi-square test was used to compare qualitative variables between the two groups. SPSS v. 20 was used for statistical analysis.

Results: The most frequent age range of diabetic children was 12 - 14 years (55\%) and 15 - 19 years in the experimental and control groups (65\%), respectively. Most fathers and mothers were self-employed (60\%) and homemakers (85\%), respectively. The mean score of burden of care imposed on families before the intervention in the experimental and control groups was 26.6 and 30.15 , respectively, that reduced to 21.6 and 30.35 three months after the intervention, respectively, showing no change.

Conclusions: Family psychoeducation can reduce family burden of care imposed by adolescents suffering from type I diabetes and increase the period for families facing the disease. It also reduces medical treatment costs and length of stay in health centers and increases quality of home care.
\end{abstract}

Keywords: Family Psychoeducation, Cost of Illness, Adolescent, Type Diabetes Mellitus

\section{Background}

Diabetes mellitus is a chronic and progressive disease that can significantly affect the life of individuals in any age (1). 3.7\% per 100000 cases suffer annually from diabetes mellitus in Iran. Currently, above 8\% (about 5 million individuals) of Iranian population suffer from diabetes (2). The disease can occur in any age but it is most prevalent in age of 10 - 15 years. In 75\% of cases, it is diagnosed in adolescence before age of 18 (3). Diabetes mellitus is an endocrine disorder that is prevalent in children. One child below 18 years old suffers from diabetes per 300-500 children (4). Some costs are incurred on the health system and society. Direct costs include costs of patient's hospitalization and care. Indirect costs involve income reduction, personal abilities decrease, long-term inability, and early death (5). Global burden of noncontagious diseases such as diabetes is considerably increasing. The disease is projected to include $60 \%$ of total burden of diseases in 2020 (6). Control of chronic diseases such as diabetes will lead individuals and family to a healthy life style, and family has an important role to play in this regard (7). Family members play active roles in activities such as blood sugar control, foot care of diabetic patient, and recognition of symptoms of blood sugar reductions (8). Family is a context for control of the affected member and activities related to diabetes management such as correction of diet and physical activity. In addition, family plays several roles in creation or correction of stress in patients with diabetes (9). Families experience high stress due to long-term side effects, managerial conflicts such as diet, and poor adherence of 
patient to treatment. These are effective in treatment process and blood sugar control of patients (10). Results of the study done by Rintala et al. (2013) showed that diabetes cause fear and concern in families (11). This chronic disease brings about long-term changes, stress, and conflicts in families (12). Lack of coordination and collaboration in family increases behavioral problems and reduces quality of family performance, leading to lower level of problem solving in the family (13). These are known as burden of care resulted from disease. Burden of care is defined as physical, socio-psychological reactions of the caregiver resulted from imbalance of caring demands with other responsibilities of the caregiver, and they have associations with socio-personal roles, physical and affective status, and financial sources of caregivers (14).

Burden of family refers to problems and challenges experienced by family when encountering disease in a family member and it includes emotional problems and practical challenges (15). In the study conducted by Moor et al. (2013), high family conflicts and their effects on family dynamics, parents' stresses, and poor control of blood sugar have been reported in families who had teenagers with type I diabetes (16).

Since many socio-psychological problems of the family are resulted from lack of family and others' awareness on diabetes, it is not possible to prevent and reduce such problems without training patients and families (17). The study conducted by Shirazi et al. (2011) showed that some educational, supportive, and psychotherapeutic interventions could have significant effects on reduction of depression, anxiety, and stress in family caregivers (18). Furthermore, the study of Heydari et al. (2012) indicated that self-efficacy and self-esteem of adolescents increased after implementation of educational programs for teenagers with type I diabetes, and level of A1C hemoglobin reduced significantly in the intervention group (19). The aim of education in diabetes is to acquire knowledge and skills for better understanding and management of the disease (20). It is necessary to pay attention to either physical or socio-psychological aspects of diabetes especially by taking strategies toward disease management via interaction and collaboration between family members and the affected member (21). Results of a study conducted by Wysocki et al. in 2009 showed that behavioral familycentered interventions that were based on teamwork of family and the affected member for diabetes management had positive effects on blood sugar control (22). One of the family collaboration-based educational programs is family psychoeducation. Family psychoeducation is a systematic structure that empowers patients and family members to encounter the disease by focusing on emotional and cognitive aspects. It aims to make a relationship between pa- tient and family as well as to make them responsible for treatment process (21). The study of Ketz et al. in 2013 indicated that presenting routine care as well as family psychoeducation caused better control of blood sugar in addition to increased involvement of parents in treatment (23). Since methods of health education have been superior to primary care of patients with diabetes, family and patient psychoeducation as a tool for nurses can be an effective step toward reduction of problems in such patients (24). Concerning the important role of nurses in the foundation of family health in different aspects of physical, psychological, and spiritual health, as well as their role in proper services and reduction of costs incurred by disease on family and government, family psychoeducation via nurses is more and more important (25). Mental health nurses provide a cognitive framework for patients through conducting interventions to accept the disease; they also follow up the treatment and increase family awareness. Therefore, training family and patient via such methods will lead to proper health, better quality of life, and reduction of care costs (26). Since diabetes can affect all physical and psychological aspects of patients' life and their families, current research aimed to study aspects and outcomes of the disease to help patients and their families. Although family psychoeducation is used for patients with psychiatric disorders, researchers tried to conduct a study to determine effects of family psychoeducation on burden of care in families who had adolescents with type I diabetes. Such intervention was conducted for the first time in Iran on families who had a member with physical disease. It is hoped that results of the study settle problems of patients with diabetes and their families.

\section{Methods}

The study is a clinical trial with pretest-posttest design in which, the effect of family psychoeducation was evaluated on burden of care in families that had adolescents with type I diabetes. The study population comprised families that had adolescents with type I diabetes aged between 12 and 19 years referring to diabetes clinic of Golestan hospital and specialized clinics affiliated to Jundishapur University of Medical Sciences in Ahwaz. First, to calculate sample size, an empirical study was done on 10 families who had inclusion criteria. The sample size included the affected child and the parent who had a close relationship with the patient. Sampling was done among individuals in the society based on study goal, inclusion, and exclusion criteria. The subjects were placed randomly in the experimental (20 families) and control (20 families) groups. Inclusion criteria involved at least one-year history 
of medical diagnosis, willingness to participate in educational programs, no history of participation in any educational program, familiarity with Persian language, and living in Ahwaz due to ease of participation in educational sessions. Exclusion criteria included hospitalization of patient during the study and family absence in two or more educational sessions. The research setting was the clinic of Golestan hospital and specialized clinics affiliated to Ahwaz Jundishapur University of Medical Sciences. To collect data, two questionnaires of demographic information and diabetes family impact-scale (DFI-S) were used. The demographic information questionnaire included information related to age and gender of child and parents, education of parents, job of parents, duration of diabetes, and economic status. DFI-S has been presented by American diabetes association. The questionnaire contains 14 items scored by a Likert scale (never, sometimes, most often, and always) and it measures family burden in four areas of education, job, finance, and sense of wellbeing. Items 1 to 4,5 to 7,8 to 10 , and 11 to 14 have been designated to measure burden in terms of education, burden in terms of job, financial pressure, and burden of family in terms of sense of wellbeing, respectively. The questionnaire has been scored as follows: never $=1$, sometimes $=2$, most often $=3$ and always $=4$. The total score obtained by 14 items suggests burden of care incurred on the family. The higher the score, the higher the burden of care. The questionnaire showed high internal consistency coefficient in the study of Ketz et al. (Cronbach's alpha $=0.84$ ). In the current research, CVR was used to measure validity of DFI-S (0.8) indicating that the questionnaire had a high content validity. In addition, Cronbach's alpha was used to measure reliability of the questionnaire, showing a very good reliability $(\alpha=0.85)$. After taking a valid letter of recommendation, researchers referred to research setting. Then, they introduced themselves to authorities of research setting, took their agreement, and explained exactly the objectives. The study was conducted after obtaining informed consent and trust of subjects under study. After dividing subjects into two groups, demographic information was collected and burden of care experienced by families was measured by DFI-S. Family psychoeducation was held for the experimental group within 8 sessions of 90 minutes twice a week. The control group received no educational intervention except for routine care. Family psychoeducation was started by familiarizing the members with each other, describing group work, and time duration of sessions. Sessions were as follows:

Session 1: introduction of individuals to each other, description of educational goals and sessions, role of family in disease management, adolescents with diabetes and self-care, teamwork and interaction between family and patient in disease control.

Session 2: disease control via family and patient, psychological conflicts and problems of family and patient, how to prevent conflicts created during disease control and management using coping and problem solving strategies.

Session 3: risks of disease and its nature, side effects of disease in long-term and challenges encountered by family and adolescents with diabetes when controlling and managing the disease.

Session 4: skills required for diabetes control and management via involvement of family and self-care activities of adolescents, how to support adolescents with diabetes for self-care.

Session 5: disease control and management via blood sugar monitoring, how to control blood sugar at the moment, self-care of adolescents with diabetes during blood sugar monitoring, family support of adolescents and their interaction in this regard.

Session 6: disease control and management via consideration of medicinal regimen, how to use insulin at the moment, self-care of adolescents with diabetes via injection of insulin, family support of adolescents and their interaction, team work of family and adolescents regarding medicinal regimen.

Session 7: disease control and management with consideration of diet and physical activity, how to consider diet and physical activity at the moment, self-care of adolescents with diabetes via consideration of diet and physical activity, family support of adolescents and their interaction, team work of family in this regard.

Session 8: summarization of materials and description of family's opinions about the sessions

Each session of family psychoeducation was held as follows: introduction and start of the session, introducing the subject in each session (10 minutes), describing materials via conductor of educational session (30 minutes), and how to solve problems and manage disease via family teamwork and problem solving methods (20 minutes). Both groups recompleted DFI-S three months after the educational sessions. Data obtained from the experimental and control groups were statistically analyzed. Descriptive and analytical statistics were used to analyze data. Descriptive results were shown in tables as frequency and percentage. To compare the mean score of family burden of care, independent and paired $\mathrm{T}$ tests were used. To compare the mean score of abnormal data, Mann-Whitney test was used after administering Kolmogorov-Smirnov test to measure data normalization. Cohen's $d$ index was used to measure the effect size. To compare qualitative variables between the groups, Chi-square test was used considering significance level of 0.05 . SPSS version 20 was used to conduct sta- 
tistical analyses. All ethical considerations were observed in the research and ethical code IR.ajums.rec.1394457 was received from the ethics committee of research and technology development assistance of Ahvaz Jundishapur University of Medical Sciences.

\section{Results}

According to the results in Table 1, 55\% of the adolescents with diabetes in the experimental group were between 12 and 14 years old and 65\% of the adolescents with diabetes in the control group were between 15 and 19 years old (as the highest frequencies). Chi-square showed no significant difference between the experimental and control groups regarding mean age; thus both groups were the same $(\mathrm{P}=0.2)$.

According to the results in Table 1, 55\% of the adolescents with diabetes in the experimental group were middle school students while they were equally distributed in the educational levels in the control group (50\%). Chisquare showed no significant difference between the experimental and control groups regarding education; thus both groups were the same in terms of education $(\mathrm{P}=0.75)$.

According to the results in Table $1,60 \%$ of fathers were self-employed and $85 \%$ of mothers were homemakers (the highest frequencies). Chi-square showed no statistically significant difference in job status of fathers $(\mathrm{P}=0.79)$ and mothers $(P=0.23)$.

According to the results in Table 1, most participants in the experimental (70\%) and control (65\%) groups had monthly income above one million Tomans. Chi-square showed no significant difference between the experimental and control groups regarding monthly income ( $\mathrm{P}=$ 0.92).

According to the results in Table 2, before intervention, the mean score of burden of care incurred on families in the experimental group was 26.6 and it was 30.91 in the control group. Mann-Whitney test showed no statistically significant difference between the groups $(\mathrm{P}=0.17)$.

According to the results in Table 2, three months after intervention, the mean score of burden of care incurred on families in the experimental group was 21.6 and it was 30.35 in the control group. T test showed a statistically significant difference between the groups $(\mathrm{P}=0.0001)$, suggesting a change in the mean score of burden of care incurred on families. Furthermore, by obtaining effect size of 0.799, it can be concluded that burden of care is very different between the groups, suggesting positive and significant effect of intervention.

\section{Discussion}

Although there are several challenges to control diabetes in adolescents, this disease can be well managed by family support (27). Concerning multidimensionality of burden of care on the family and the effect of different variables, some variables such as job of parents, age of teenagers, and monthly income of parents were compared in both groups that showed no significant difference. Therefore, both groups were similar regarding above variables and the effect of the variables was controlled. The results of the study indicated that three months after administering family psychoeducation to subjects under study, burden of care incurred on families reduced significantly, suggesting the positive effect of family psychoeducation on families with a type I diabetic child. Most researchers believe that family burden of care is a multidimensional concept because some problems and challenges are experienced by family and the patient such as financial problems, the time spent by caregivers, disturbance in daily roles, and socio-psychological stress (28). However, social support including family support can help improve the patient by reducing stress, changing condition of disease, increasing satisfaction, and decreasing unhealthy behaviors (29). The family can affect chronic diseases such as diabetes. The family is effective on chronic diseases in two ways: first, emotional climate of the family that has a direct effect on physiology of patient and second, family involvement in disease problems that improves selfcare behaviors (30). The study done by Muller et al. (2009) indicated that after giving responsibility to adolescents with type I diabetes, quality of life increased significantly in all ages of adolescence. Furthermore, parents showed reduced stress induced by hypoglycemia and problems of nutritional management (31). Since quality of life of adolescents with diabetes increased by blood sugar control and parents experienced reduced stress, family burden of care decreased, as a result. The result is consistent with the findings of the present study. Much research has addressed blood sugar control and self-care behaviors of adolescents with diabetes via different interventions; however, family, its problems, family interaction with the diabetic patient, and teamwork have been less paid attention to while they play important roles in control and management of diabetes. Therefore, family problems and interaction between family and patient as a teamwork should be paid attention to. Family psychoeducation gives opportunities to families to identify challenges when taking care of their children and use capabilities of themselves and their family members to cope with challenges. The study of Mayberry et al. (2012) showed that social support had a direct effect on selfcare behaviors of diabetic patients and it had indirect ef- 
Table 1. Frequency and Percentage Distribution of Demographic Characteristics of Subjects in Both the Intervention and Control Groups

\begin{tabular}{|c|c|c|c|c|c|}
\hline \multirow[t]{2}{*}{ Variable } & \multicolumn{2}{|c|}{ Experimental } & \multicolumn{2}{|c|}{ Control } & \multirow[t]{2}{*}{ P Value } \\
\hline & Frequency & Percentage & Frequency & Percentage & \\
\hline Adolescent Age & & & & & 0.2 \\
\hline $12-14$ & 11 & 55 & 7 & 35 & \\
\hline$>15$ & 45 & 9 & 13 & 65 & \\
\hline Education & & & & & 0.75 \\
\hline Secondary school & 11 & 55 & 10 & 50 & \\
\hline High school & 9 & 45 & 10 & 50 & \\
\hline Father's job & & & & & 0.79 \\
\hline Self employed & 10 & 50 & 12 & 60 & \\
\hline Employee & 10 & 50 & 8 & 40 & \\
\hline Mother's job & & & & & 0.23 \\
\hline Housewife & 14 & 70 & 17 & 85 & \\
\hline Employed & 6 & 30 & 3 & 15 & \\
\hline Frequency & 20 & & & 20 & \\
\hline
\end{tabular}

Table 2. Comparison of Means Scores of Burden of Care in the Experimental and Control Groups Before and After the Intervention with 3 Months Fallow-up

\begin{tabular}{l|c|c|c|c|c}
\hline Study Group & $\begin{array}{c}\text { Experimental } \\
\text { Mean } \pm \text { SD }\end{array}$ & $\begin{array}{c}\text { Control } \\
\text { Mean } \pm \text { SD }\end{array}$ & PValue & D - Index \\
\hline Before intervention & $26.6 \pm 6.7$ & $30.91 \pm 8.3$ & 0.17 & 2.66 \\
\hline After intervention & $21.6 \pm 3.37$ & $30.35 \pm 1.66$ & 0.0001 & 0.799 \\
\hline
\end{tabular}

fect on blood sugar control of patients with diabetes (32). According to several studies, family psychoeducation not only reduces burden of care, but also affects positively the attitude of family members as caregivers (33). The study of Ketz et al. (2014) indicated that family psychoeducation caused better control of blood sugar, higher involvement, and more attention of family members to patient with diabetes. The result is consistent with that of the current study (21). The strength of the study of Ketz (compared to the current study) is larger sample size and follow-up of subjects under study for two years that showed the effect of intervention in long-term. In addition, the study of Ekinici and Tanriverdi (2012) showed that family psychoeducation could lead to the reduction of burden of care on families with a patient. The result is in agreement with the findings of the current study (30). Although family psychoeducation has been implemented for patients with psychological disorders, the implementation of family psychoeducation for families with a member with chronic physical disease provides a condition for families to recognize psychological problems related to control of diabetes, perceive the role of patient in disease control, work together, and interact as a team to control and manage problems. These are positive outcomes of paying attention to familycentered interventions, which showed positive effects on psychological processes of families in the present study.

\subsection{Conclusion}

Based on the results of the study, family psychoeducation can reduce burden of care in families with type I diabetic adolescents by increasing coping strategies in families when encountering the disease. This therapeutic method reduces treatment and hospitalization costs and increases quality of home care. The results of the study can create further research ideas in minds of researchers.

\section{Acknowledgments}

The present thesis was funded by the approved projects No. D-9412. All rights of this thesis are reserved for the vice chancellor of research of Ahwaz University of Medical Sciences. The authors sincerely express their gratitude to the vice chancellor of research of Ahwaz University of Medical 
Survey files of adolescents with type 1 diabetes in

Golestan hospital diabetes clinic and specialized clinic affiliated of the Ahvaz

Jundishapur University of medical sciences and a pilot study on 10 families with

adolescents with type 1 diabetes aged between 12 and 19 years



Completing the burden of family caregiving questionnaire (DFI-S) by the subjects after three months

Statistical analysis of data collected

from two study groups

Sciences for funding this project. This study has been registered in the Iranian registry of clinical trials with the code “IRCT2015121525532N1.”

\section{References}

1. Ellis M, Jayarajah C. Adolescents' view and experiences of living with type 1 diabetes. Nurs Child Young People. 2016;28(6):28-34. doi: 10.7748/ncyp.2016.e727. [PubMed: 27387634].

2. Salehi S, Shafiei S, Amini P, Abdeyazdan G. Self-care performance in children and adolescents with type 1 diabetes mellitus. JJ Chronic Dis Care. 2014;3(3).

3. Hockenberry MJ, Wilson D. Wong's nursing care of infants and children. Elsevier Health Sciences; 2014. 
4. Mendenhall E, Norris SA, Shidhaye R, Prabhakaran D. Depression and type 2 diabetes in low- and middle-income countries: a systematic review. Diabetes Res Clin Pract. 2014;103(2):276-85. doi 10.1016/j.diabres.2014.01.001. [PubMed: 24485858].

5. Giorda CB, Manicardi V, Diago Cabezudo J. The impact of diabetes mellitus on healthcare costs in Italy. Expert Rev Pharmacoecon Outcomes Res. 2011;11(6):709-19. doi: 10.1586/erp.11.78. [PubMed: 22098288].

6. Saidi O, O'Flaherty M, Mansour NB, Aissi W, Lassoued O, Capewell S, et al. Forecasting Tunisian type 2 diabetes prevalence to 2027: validation of a simple model. BMC Public Health. 2015;15:104. doi: 10.1186/s12889015-1416-z. [PubMed: 25885910].

7. Arends-Toth J, Van de Vijver FJ. Family relationships among immigrants and majority members in the Netherlands: The role of acculturation. Applied Psychology. 2008;57(3):466-87.

8. Paddison C. Family support and conflict among adults with type 2 diabetes. Europ Diabet Nurs. 2010;7(1):29-33.

9. Cole I, Chesla CA. Interventions for the family with diabetes. Nurs Clin North Am. 2006;41(4):625-39. doi: 10.1016/j.cnur.2006.07.001. [PubMed: 17059978] vii.

10. Tsiouli E, Alexopoulos EC, Stefanaki C, Darviri C, Chrousos GP. Effects of diabetes-related family stress on glycemic control in young patients with type 1 diabetes: Systematic review. Can Fam Physician. 2013;59(2):143-9. [PubMed: 23418238].

11. Rintala TM, Jaatinen P, Paavilainen E, Astedt-Kurki P. Interrelation between adult persons with diabetes and their family: a systematic review of the literature. J Fam Nurs. 2013;19(1):3-28. doi: 10.1177/1074840712471899. [PubMed: 23288886].

12. Pereira MG, Berg-Cross L, Almeida P, Machado JC. Impact of family environment and support on adherence, metabolic control, and quality of life in adolescents with diabetes. Int J Behav Med. 2008;15(3):187-93. doi: 10.1080/10705500802222436. [PubMed: 18696312].

13. Dashtbozorgi B, Ghadirian F, Khajeddin N, Karami K. Effect of family psychoeducation on the level of adaptation and improvement of patients with mood disorders. Iran J Psychiat Clinical Psychol. 2009;15(2):193-200.

14. Valizade L, Jvnbkhsh F, Pashaie S. Once parents caring for children with cancer at Children's Hospital of Tabriz. J Clinical Nurs Midwifery. 2014;3:13-20.

15. Ennis E, Bunting BP. Family burden, family health and personal mental health. BMC Public Health. 2013;13:255. doi: 10.1186/1471-2458-13-255. [PubMed: 23517472].

16. Moore SM, Hackworth NJ, Hamilton VE, Northam EP, Cameron FJ. Adolescents with type 1 diabetes: parental perceptions of child health and family functioning and their relationship to adolescent metabolic control. Health Qual Life Outcomes. 2013;11:50. doi: 10.1186/1477-7525-1150. [PubMed: 23521786].

17. Al-Shahrani AM, Hassan A, Al-Rubeaan KA, Al Sharqawi AH, Ahmad NA Effects of diabetes education program on metabolic control among Saudi type 2 diabetic patients. 2012

18. Shirazi M, Anoosheh M, Rajab A. The effect of self care program education by group discussion method on self concept in diabetic adolescent girls. Iran Diabet Society. 2011

19. Heidari M, Alhani F, Kazemnejid A, Tol A, Moezi F. Assessing the effect of educational program based on empowerment model on HbA1C among adolescents with type 1 diabetes. 2012

20. Berry E, Lockhart S, Davies M, Lindsay JR, Dempster M. Diabetes distress: understanding the hidden struggles of living with diabetes and exploring intervention strategies. Postgrad Med J. 2015;91(1075):27883. doi: 10.1136/postgradmedj-2014-133017. [PubMed: 25827438].

21. Rummel-Kluge C, Kissling W. Psychoeducation for patients with schizophrenia and their families. Expert Rev Neurother. 2008;8(7):1067-77. doi: 10.1586/14737175.8.7.1067. [PubMed: 18590477].

22. Wysocki T, Nansel TR, Holmbeck GN, Chen R, Laffel L, Anderson $B J$, et al. Collaborative involvement of primary and secondary caregivers: associations with youths' diabetes outcomes. J Pediatr Psychol. 2009;34(8):869-81. doi: 10.1093/jpepsy/jsn136. [PubMed: 19112077].

23. Katz ML, Volkening LK, Butler DA, Anderson BJ, Laffel LM. Familybased psychoeducation and Care Ambassador intervention to improve glycemic control in youth with type 1 diabetes: a randomized trial. Pediatr Diabetes. 2014;15(2):142-50. doi: 10.1111/pedi.12065. [PubMed: 23914987].

24. Aalaa M, Malazy OT, Sanjari M, Peimani M, Mohajeri-Tehrani M. Nurses' role in diabetic foot prevention and care; a review. J Diabetes Metab Disord. 2012;11(1):24. doi: 10.1186/2251-6581-11-24. [PubMed: 23497582].

25. Yamani N, Taleqani F. Family nursing curriculum, a step toward the development of nurse's role in Iran. Iran J Med Edu. 2012;11(9):1385-94.

26. Shin SK, Lukens EP. Effects of psychoeducation for Korean Americans with chronic mental illness. Psychiatr Serv. 2002;53(9):1125-31. doi: 10.1176/appi.ps.53.9.1125. [PubMed: 12221311].

27. Borus JS, Laffel L. Adherence challenges in the management of type 1 diabetes in adolescents: prevention and intervention. Curr Opin Pediatr. 2010;22(4):405-11. doi: 10.1097/MOP.0b013e32833a46a7. [PubMed: 20489639].

28. van der Sanden RL, Stutterheim SE, Pryor JB, Kok G, Bos AE. Coping with stigma by association and family burden among family members of people with mental illness. J Nerv Ment Dis. 2014;202(10):710-7. doi: 10.1097/NMD.0000000000000189. [PubMed: 25198703].

29. Miller TA, Dimatteo MR. Importance of family/social support and impact on adherence to diabetic therapy. Diabetes Metab Syndr Obes. 2013;6:421-6. doi: 10.2147/DMSO.S36368. [PubMed: 24232691].

30. Hartmann M, Bazner E, Wild B, Eisler I, Herzog W. Effects of interventions involving the family in the treatment of adult patients with chronic physical diseases: a meta-analysis. Psychother Psychosom. 2010;79(3):136-48. doi: 10.1159/000286958. [PubMed: 20185970].

31. Muller-Godeffroy E, Treichel S, Wagner VM, German Working Group for Paediatric Pump T. Investigation of quality of life and family burden issues during insulin pump therapy in children with Type 1 diabetes mellitus-a large-scale multicentre pilot study. Diabet Med. 2009;26(5):493-501. doi: 10.1111/j.1464-5491.2009.02707.x. [PubMed: 19646189].

32. Mayberry LS, Osborn CY. Family support, medication adherence, and glycemic control among adults with type 2 diabetes. Diabetes Care. 2012;35(6):1239-45. doi: 10.2337/dc11-2103. [PubMed: 22538012].

33. Tanriverdi D, Ekinci M. The effect psychoeducation intervention has on the caregiving burden of caregivers for schizophrenic patients in Turkey. Int J Nurs Pract. 2012;18(3):281-8. doi: 10.1111/j.1440172X.2012.02033.x. [PubMed: 22621299]. 